\title{
Cellulase Inhibits Burkholderia cepacia Biofilms on Diverse Prosthetic Materials
}

\author{
SATISH KUMAR RAJASEKHARAN and SAMIRAJ RAMESH*
}

Department of Microbiology, PRIST University, Thanjavur, Tamil Nadu, India

Submitted 21 December 2012, revised 26 March 2013, accepted 15 July 2013

\begin{abstract}
Burkholderia cepacia is an opportunistic pathogen causing infections in patients with cystic fibrosis. Patients with implanted devices are prone to B. cepacia infections due to its ability to grow as biofilms. Knowing the importance of polysaccharides in a biofilm, enzymes that degrade them were targeted as a possible candidate for antibiofilm agents. In this study, the antibiofilm potential of cellulase against $B$. cepacia biofilms formed on various prosthetic materials was tested. Cellulase exhibited significant antibiofilm activity against $B$. cepacia without having much action on its growth, thus ruling out the chance of selection pressure and subsequent development resistance.
\end{abstract}

Key words: Burkholderia cepacia, anti-biofilm activity, cellulase, prosthetic materials

Burkholderia cepacia is an imperative pathogen known to accentuate the pathogenesis of cystic fibrosis (CF) and chronic granulomatous diseases in immunocompromised individuals. The opportunistic human pathogen, grouped under B. cepacia complex (BCC) is frequently retrieved from CF patients (McDowell et al., 2001). The B. cepacia infections predominantly result in asymptomatic carriage, gradual decline in lung function and cepacia syndrome, a terminal necrotizing pneumonia (Vandamme, 2001). The sternness of these infections in immunocompromised hosts is attributed to the organism's diverse virulence factors and the ability to form biofilms. B. cepacia secretes numerous virulence factors viz. lipase, metalloproteases, serine proteases, which intensify the pathogenic traits via cep quorum sensing system (Shawn et al., 1999). B. cepacia is also known to produce exopolysaccharide cepacian that reinforce the biofilm integrity (Monica et al., 2004). The bacteria within the biofilm consortium are highly resistant to standard antibiotics. The innate and emerging resistance of biofilm residing bacteria against antibiotics in the current scenario consequently lead to the screening of various alternative sources to eradicate the bacterial pathogen (Jones et al., 2004). Cellulase cleaves the $\beta-1,4$ linkages in exopolysaccharides, a major constituent of biofilms. Studies on the use of cellulase to inhibit biofilm have been reported and describe partial inhibition of Pseudomonas aeruginosa biofilms
(Melanie etal., 2003). Other studies have reported the accumulation of BCC on various contaminated pharmaceuticals and cosmetics (Sousa et al., 2011). Hence, the present study has focused on inhibition of B. cepacia biofilms on prosthetic materials like poly styrene, polyvinyl, polycarbonate and glass surfaces using cellulase enzyme.

Reference strains of B. cepacia (MTCC-1617, 6902 and 438) and B. cepacia (EU053863.1) were initially used and later the strain 1617 was used for all the assays due to its clinical origin and superior biofilm forming ability. Cellulase from Aspergillus niger (C1184-SigmaAldrich) and partially purified cellulase from Bacillus sp. DGV19 (EU053862.1) were used for quantitative biofilm inhibition assays. A fixed concentration of cellulase (100 units $\mathrm{ml}^{-1}$ ) was used for all the analysis. Cellulase production in cell free supernatant (CFS) of Bacillus sp. DGV19 was phenotypically confirmed by carboxymethyl cellulose clear zone (CMCZ) test and by the indication of an evident $76 \mathrm{kDa}$ protein band in SDS-PAGE gel stained with comassive brilliant blue (CBB R250). For biofilm assays, sterile disks of $10 \mathrm{~mm}$ diameters of polystyrene (Tarsons Product Pvt. Ltd., India), glass slides, polyvinyl chloride (PVC) and polycarbonate (Himedia., India) were place on the bottom of a 24 -well microtiter plate. The $100 \mu$ of the enzyme was added to nutrient broth supplemented with $0.5 \%$ glucose containing the bacterial suspension

* Corresponding author: S. Ramesh, Dept. of Microbiology, PRIST University, Thanjavur - 614 904, Tamil Nadu, India; phone: +91 99761 93870; fax: 04362 - 265150; e-mail: marineramesh2020@gmail.com 

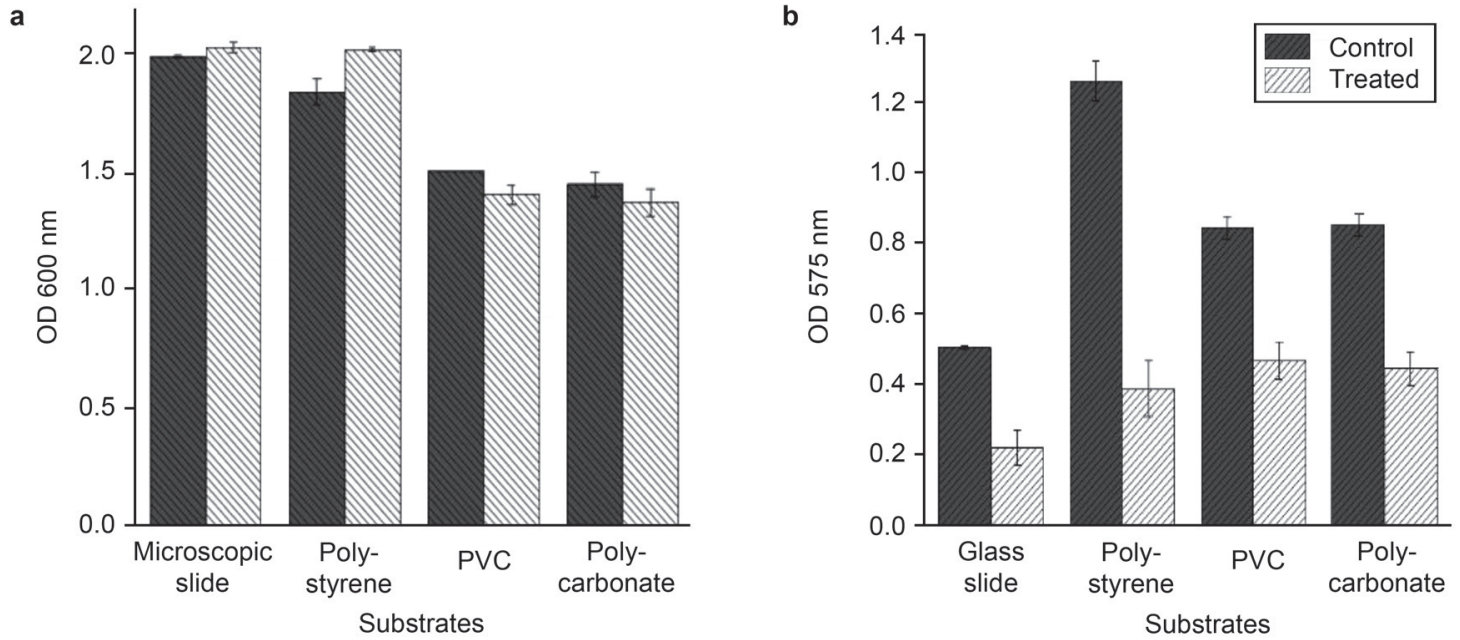

Fig. 1. Effect of cellulase on (a) growth and (b) biofilm formation of B. cepacia grown on different diverse prosthetic materials.
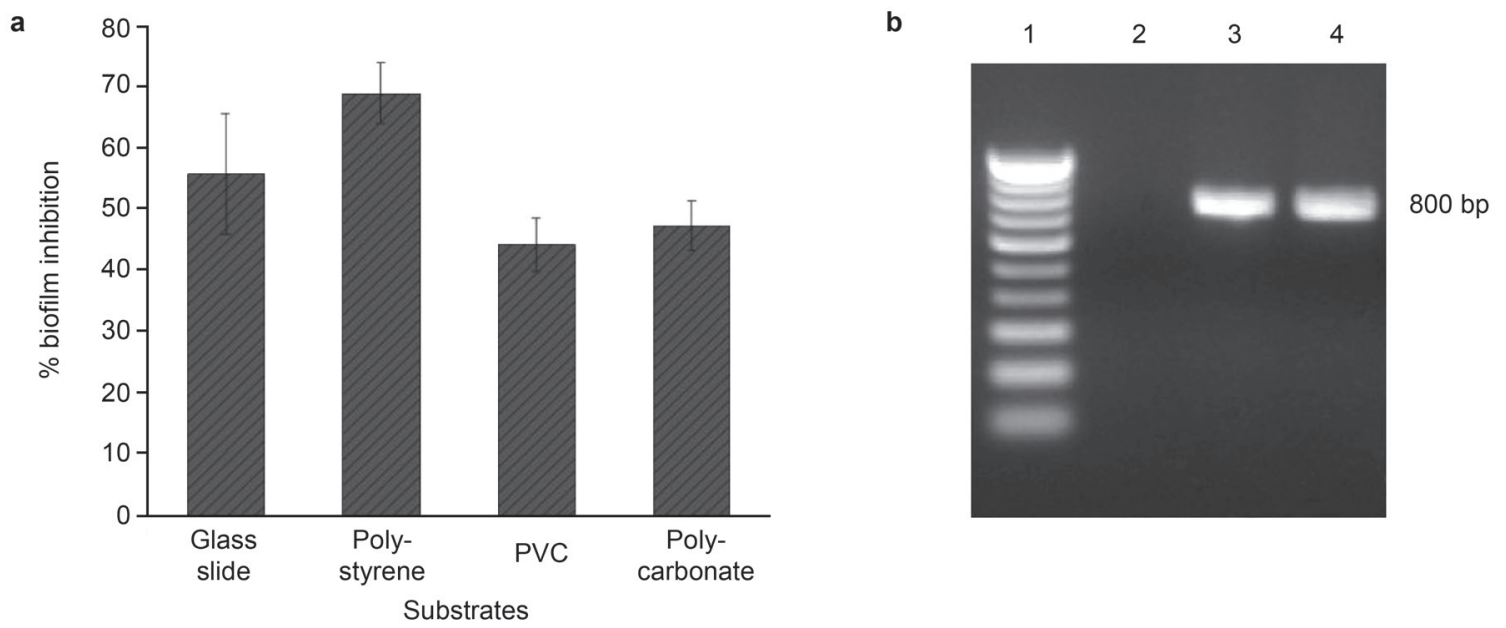

Fig. 2. (a) Influence of cellulase on B. cepacia biofilms on various prosthetic materials. (b) PCR amplification of aiiA gene. Lane 1: 100 bp DNA ladder. Lane 2: Negative control and Lane 3 \& 4: 800 bp aiiA gene fragment from Bacillus sp. DGV19.

at $10^{6} \mathrm{CFU} \mathrm{ml} l^{-1}$ and incubated at $37^{\circ} \mathrm{C}$ for 24 hours. Following incubation, the plates were washed with distilled water to remove the unbound bacterial cells and the biofilm was stained with $0.4 \%$ crystal violet. The substrates were destained with $95 \%$ ethanol and biofilm density was quantified at $575 \mathrm{~nm}$ using UV spectrophotometer (Bakkiyaraj et al., 2010).The bacteriostatic effect of cellulase in broth cultures were quantitatively measured at $600 \mathrm{~nm}$. Similar sets of experiments were conducted with CFS of Bacillus sp. DGV19. All the experiments were done in triplicates and the statistical analyses were executed using SPSS. Values were expressed as mean \pm SD and the statistical significance was analyzed by student's t-test. The percentage of biofilm inhibition was estimated by the following formula; $\%$ biofilms inhibition $=([$ control OD $575 \mathrm{~nm}-$ test OD $575 \mathrm{~nm}] /$ control OD $575 \mathrm{~nm}$ ) x 100.

Cellulase had no effect on the growth of B. cepacia (Fig. 1a), but showed significant reduction of biofilms on various materials tested $(\mathrm{P}<0.05)$ (Fig. 1b). The biofilms on various substrates were initially examined with a light microscope and further qualitatively confirmed using a confocal laser scanning microscope (CLSM) (Zeiss/LSM 710, Carl Zeiss, Germany) after subsequent staining with $0.01 \%$ acridine orange (Bakkiyaraj et al., 2012). Focus stacking was executed using Zen 2009 software. Maximum biofilm production and subsequent inhibition by cellulase/CFS was visualized in polystyrene surfaces when compared to the other substrates (Fig. 2a). The 3-dimensional CLSM images showed considerable reduction in B. cepacia biofilm density and thickness when treated with cellulase (Fig. 3). Focus stacking revealed approximately 50\% diminution of biofilms formed by B. cepacia on various substrates tested. Maximum inhibition was eminent in polystyrene surfaces with $68 \%$, followed by $57 \%$ on glass. 50\% inhibition was observed on PVC and polycarbonate surfaces respectively. The CFS from Bacillus 


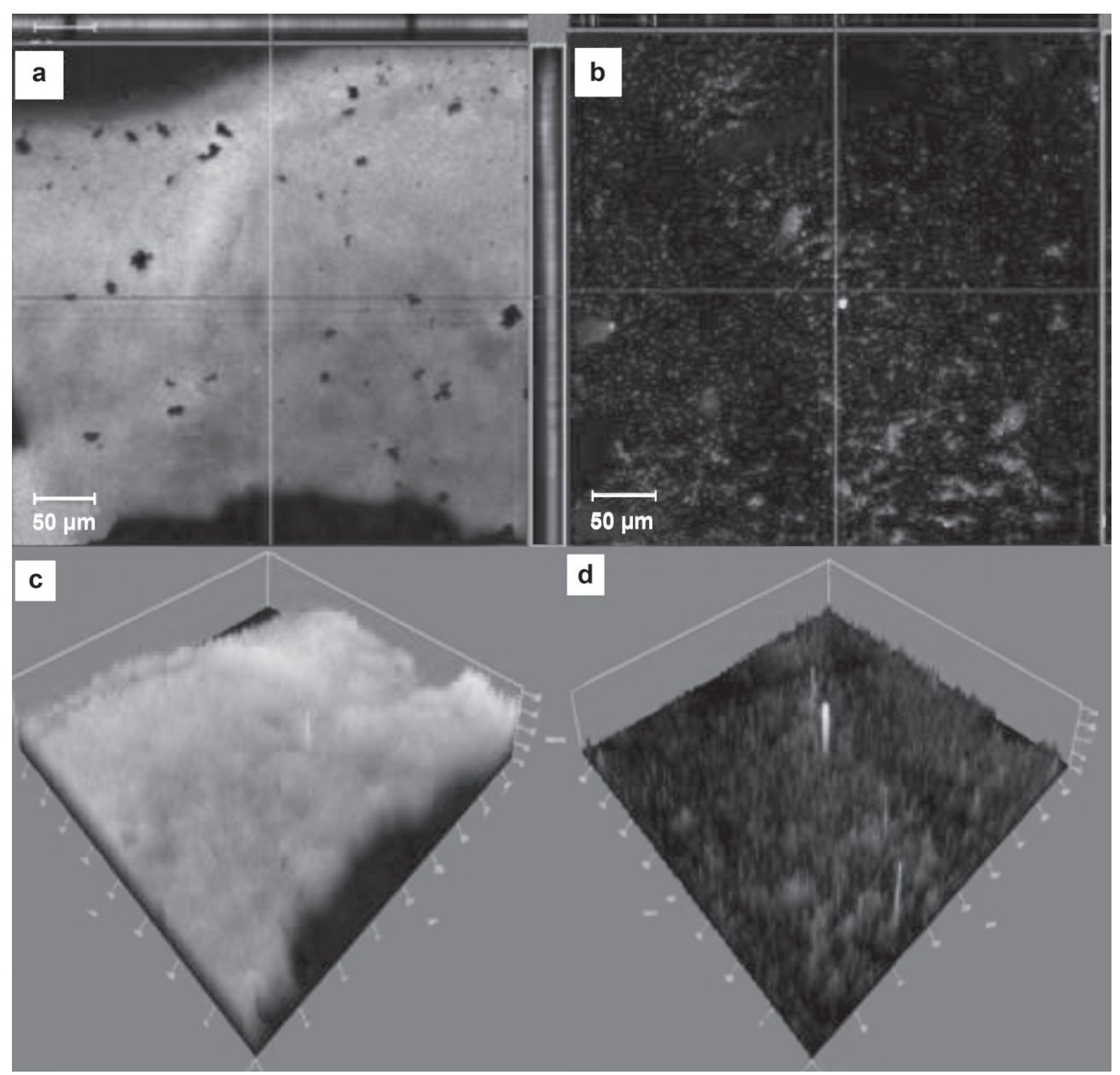

Fig. 3. Representative 3D CLSM images of biofilms formed by B. cepacia. (a) Ortho and (c) $2.5 \mathrm{~d}$ images of control B. cepacia biofilms. (b) Ortho and

(d) $2.5 \mathrm{~d}$ images of B. cepacia biofilms treated with cellulase enzyme.

sp. DGV19 produced cellulase, which possessed antibiofilm activity against the opportunistic pathogen. The inhibition of B. cepacia biofilms by CFS was not exclusively due to the enzymatic activity of cellulase, as Bacillus sp. DGV19 was also shown to possess quorum quenching (QQ) activity against the indicator strain, Chromobacterium violaceum ATCC12472. The presence of aiiA homologue gene, encoding $N$-Acyl-homoserine lactonase in Bacillus sp. DGV19 was confirmed by PCR amplification using forward (5'ATGGGATCCATGACAGTAAAGAAGCTTTAT-3') and reverse (5'-GTCGAATTCCTCAACAAGATACTCCTAATG-3') primers (Fig. 2b).

In conclusion, the results of the present study apparently manifest the ability of cellulase to marginally inhibit B. cepacia biofilms grown on various prosthetic materials. It has also been demonstrated that the cellulase does not inhibit the growth of the bacteria while controlling its biofilm formation on different substrates. The plausible mechanism of biofilm inhibition may involve the degradation of exopolysaccharide cepacian by the cellulase. Further study is warranted for imple- menting the enzyme treatment in combination with other anti-quorum sensing agents/enzymes for absolute inhibition of $B$. cepacia biofilms on clinical devices.

\section{Acknowledgments}

The authors are grateful to the authorities of PRIST University for the facilities. Financial support rendered to Satish Kumar Rajasekharan in the form of Junior Research Fellow from DRDO, GoI (ERIP/ER/1103948/M/01/1377) has been thankfully acknowledged.

\section{Literature}

Bakkiyaraj D. and S.K. Pandian. 2010. In vitro and in vivo antibiofilm activity of a coral associated actinomycete against drug resistant Staphylococcus aureus biofilms. Biofouling. 26: 711-717.

Bakkiyaraj D., C. Sivasankar and S.K. Pandian. 2012. Inhibition of quorum sensing regulated biofilm formation in Serratia marcescens causing nosocomial infections. Bioorg. Med. Chem. Lett. 22: 3089-94. Jones A.M., M.E. Dodd and A.K. Webb. 2004. Burkholderia cepacia: current clinical issues, environmental controversies, and ethical dilemnas. Eur. Respir. J. 17: 295-301.

McDowell A.A., E. Mahenthiralingam and J.E. Moore. 2001. PCRbased detection and identification of Burkholderia cepacia complex 
pathogens in sputum from cystic fibrosis patients. J. Clin. Microbiol. 39: 4247-4255.

Melanie L. and W.A. Kimberly. 2003. The use of cellulase in inhibiting biofilm formation from organisms commonly found on medical implants. Biofouling. 19: 77-85.

Monica V.C., A.S. Silvia and H.L. Jorge. 2004. Studies on the involvement of the exo-polysaccharide produced by cystic fibrosis-associated isolates of Burkholderia cepacia complex in biofilm formation and in persistence of respiratory infection. J. Microbiol. 42: 3052-3058.
Shawn L., C. Barbara and E.P. Greenberg. 1999. Quorum Sensing in Burkholderia cepacia: Identification of the LuxRI Homologs CepRI. J. Bacteriol. 181: 748-756.

Sousa S.A., C.G. Ramos and J.H. Leitao. 2011. Burkholderia cepacia complex: Emerging multi host pathogens equipped with a wide range of virulence factors and determinants. Int. J. Microbiol. doi:10.1155/2011/607575.

Vandamme P. 2001. Burkholderia cepacia: Pandora's Box redefined. BCCM. News. 9: 2-3. 